\title{
Analysis of Ares 1 Ascent Navigation Options
}

\author{
Lee Norris ${ }^{1}$, Yee-Chee $\mathrm{TaO}^{2}$ and Robert Hall ${ }^{3}$ \\ C. S. Draper Laboratory, Cambridge, MA 02139 \\ and \\ Jason Chuang $^{4}$ and Mark Whorton ${ }^{5}$ \\ Marshall Space Flight Center, Huntsville, AL 35812
}

$\begin{array}{ll}\omega^{\mathrm{B}} & =\text { Body angular rate } \\ \mathrm{i} & =\text { Inclination } \\ \text { IMU } & =\text { Inertial Measurement Unit } \\ \text { MECO } & =\text { Main Engine Cutoff } \\ \text { MSFC } & =\text { Marshall Space Flight Center } \\ \text { KSC } & =\text { Kennedy Space Center } \\ \text { ppm } & =\text { Parts Per Million } \\ \text { SF } & =\text { Specific force } \\ \text { ZUPT } & =\text { Zero Velocity Update }\end{array}$

\section{Nomenclature}

\section{Introduction}

$\mathrm{T}$ HE paper documents a collaborative analysis of ascent Navigation options for the Ares 1 launch vehicle by the NASA Marshall Space Flight Center (MSFC) and the C. S. Draper Laboratory. The objective of the work was the development of a Navigation concept and supporting requirements which meet the Ares 1 accuracy specification in a manner which is straightforward, reliable, and cost effective. Six primary Navigation architectures were considered. In each case analysis was performed to determine under what conditions the required accuracy at second stage cutoff could be achieved. Those architectures which met the accuracy requirements were then assessed in terms of cost, complexity, and reliability to determine a baseline Navigation approach and the primary supporting requirements.

\section{The Ares 1 Launch Vehicle}

The Ares 1 launch vehicle currently under development by NASA is responsible for lifting the Orion capsule and its crew into orbit. The vehicle will consist of two stages - a 5-segment solid fuel Shuttle heritage first stage and a liquid fuel second stage incorporating an adaptation of an Apollo heritage J-2 engine. Launch will be from the Kennedy Space Center (KSC). The Ares 1 Navigation hardware will be located within an instrumentation ring near the top of the second stage and just behind the Orion capsule. The primary instrumentation will be an Inertial Measurement Unit (IMU), which will provide inertial position and velocity information at as high as $200 \mathrm{~Hz}$. The use of auxiliary instrumentation is considered in the assessment of options below. The Navigation system will be activated approximately one hour prior to launch for pre-launch initialization. The flight portion of the mission will last approximately ten minutes, with staging occurring approximately two minutes into flight. During flight, the Navigation system will provide position and velocity information to Guidance at $1 \mathrm{~Hz}$ for use in developing thrust

\footnotetext{
${ }^{1}$ Group Leader, Navigation Group; Draper Laboratory, Cambridge MA 02139: hnorris@ draper.com

${ }^{2}$ Senior Member Technical Staff ; Draper Laboratory, Cambridge MA 02139: yctao@draper.com

${ }^{3}$ Principal Member Technical Staff; Draper Laboratory, Huntsville, AL 35812: Robert.A.Hall@ nasa.gov

${ }^{4}$ Ares I Navigation Lead, EV 42, MSFC, AIAA Senior Member.

${ }^{5}$ Branch Chief, EV42/Guidance, Navigation, and Mission Analysis Branch; MSFC, AIAA Associate Fellow. 
vector commands and will provide vehicle attitude and attitude rate information to Flight Control at $50 \mathrm{~Hz}$ for use in stabilizing the vehicle while achieving the Guidance commands.

\section{Ares 1 Launch Vehicle Requirements}

The primary Ares 1 objective is to accurately place the Orion capsule on an orbital trajectory at second stage Main Engine Cutoff (MECO). Trajectory errors which exist at second stage MECO must be corrected by the Orion capsule, which in turn requires Orion to carry dedicated propellant and thus increases its mass (and decreases its payload). The second stage MECO error requirements are thus a shared burden, with the allocation of allowable error to Ares 1 representing an allocation which does not unnecessarily constrain either system. The allocation to Ares 1 must then be further partitioned between the Navigation, Guidance, and Control functions. Practically speaking and for purposes of this work, the second stage MECO requirements are effectively levied on the Navigation function as the Guidance and Control contributions to MECO error will be small.

The agreed upon second stage MECO error requirements are a semi-major axis error of $2.67 \mathrm{~nm}(16,223$ feet, one sigma) and a wedge angle error of $84 \operatorname{arcsec}(1 \mathrm{sigma})$. Of these, the wedge angle requirement would prove to be the most challenging. Wedge angle is defined as the angle between the desired and achieved orbital planes as
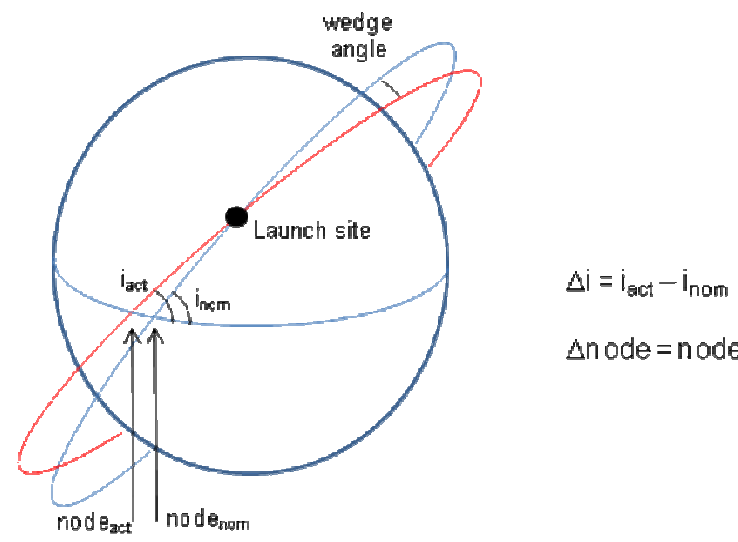

snode = node ac: - node nom

Figure 1- Wedge Angle illustrated in Figure 1 and can be determined as the angle between the angular momentum vector of the desired and achieved orbits. For an idealized vehicle in which the velocity was imparted impulsively, the wedge angle error would equal the launch azimuth error. In practice this is still a good approximation. For a launch at the equator, the wedge angle error for an impulsive vehicle would correspond to the orbit inclination error. For a nonequatorial launch, however, the wedge angle error may be decomposed into an inclination error and a node error.

In addition, the Ares 1 vehicle is required to be "Fail-Op/Fail-Safe". This

means that with a first Failure the vehicle continues to Operate normally, with a second Failure the vehicle continues to Operate safely.. The result of the requirement is that three IMUs will be carried on board such that if two fail, the third can provide safe mission abort. The presence of three IMUs in the system will motivate one of the architectural options discussed below.

\section{Ascent Reference Trajectory}

The reference trajectory used for analysis is illustrated in Figure 2. This trajectory is representative of a typical launch to an inclination of 51.6 degrees corresponding to the orbit of the International Space Station. The trajectory is based on a gravity turn first stage trajectory and a second stage trajectory based on a closed-loop Guidance algorithm such as the Powered Explicit Guidance (PEG) algorithm used by Shuttle. The first stage burn lasts for 130 seconds, achieving a maximum acceleration of $110 \mathrm{ft} / \mathrm{sec}^{2}$. The second stage burn is 460 seconds in duration and achieves a maximum acceleration of $97 \mathrm{ft} / \mathrm{sec}^{2}$. The downrange-altitude plot indicates that at staging the Ares 1 vehicle will have reached an altitude of 194,000 feet at a downrange distance of 44 nautical miles. At second stage MECO, Ares 1 will be at an altitude of 364,000 feet and 1040 nautical miles downrange. 

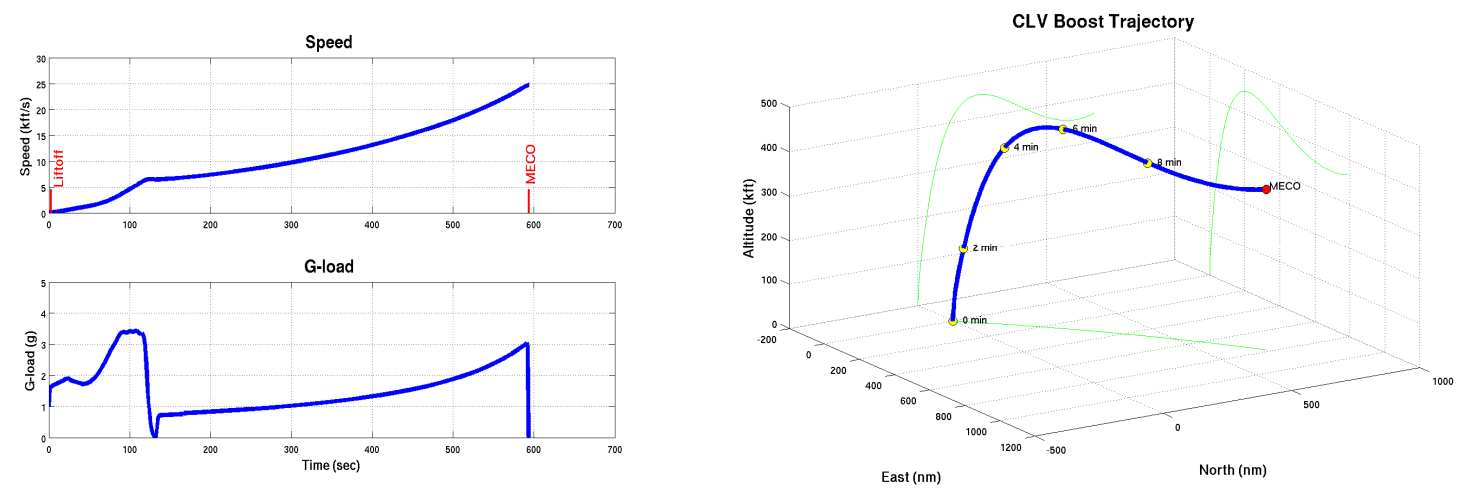

Figure 2- Trajectory Used For Analysis

\section{Error Models Used In Analysis}

MSFC established two IMU error specifications for use in analysis. The first, labeled the "Medium Quality IMU", represented an instrument package which research showed could be supplied by vendors without special considerations or procedures. The second, labeled the "High Quality IMU", corresponded to significantly higher

\begin{tabular}{|l|c|c|}
\hline Error Source & $\begin{array}{c}\text { Medium Quality } \\
\text { IMU }\end{array}$ & $\begin{array}{c}\text { High Quality } \\
\text { IMU }\end{array}$ \\
\hline Accelerometer & & \\
\hline Bias repeatability & $40 \mu \mathrm{g}$ & $20 \mu \mathrm{g}$ \\
\hline Scale factor error & $100 \mathrm{ppm}$ & $40 \mathrm{ppm}$ \\
\hline Nonorthogonality & $10 \mathrm{arcsec}$ & $2 \mathrm{arcsec}$ \\
\hline g square error & $7 \mathrm{ppm} / \mathrm{g}$ & $7 \mathrm{ppm} / \mathrm{g}$ \\
\hline g cube error & $0.1 \mathrm{ppm} / \mathrm{g} / \mathrm{g}$ & $0.1 \mathrm{ppm} / \mathrm{g} / \mathrm{g}$ \\
\hline Size effect & $2 \mathrm{mils}$ & $2 \mathrm{mils}$ \\
\hline Markov & $4 \mu \mathrm{g}, \tau=60 \mathrm{~s}$ & $2 \mu \mathrm{g}, \tau=60 \mathrm{~s}$ \\
\hline Random walk & $0.00064 \mathrm{ft} / \mathrm{s} / \mathrm{sqrt}(\mathrm{s})$ & $0.000064 \mathrm{ft} / \mathrm{s} / \mathrm{sqrt}(\mathrm{s})$ \\
\hline Gyro & & \\
\hline Bias drift & $0.006 \mathrm{deg} / \mathrm{hr}$ & $0.003 \mathrm{deg} / \mathrm{hr}$ \\
\hline Scale factor error & $5 \mathrm{ppm}$ & $1 \mathrm{ppm}$ \\
\hline Nonorthogonality & $10 \mathrm{arcsec}$ & $1.5 \mathrm{arcsec}$ \\
\hline Markov & $0.002 \mathrm{deg} / \mathrm{hr}, \tau=60 \mathrm{~s}$ & $0.001 \mathrm{deg} / \mathrm{hr}, \tau=60 \mathrm{~s}$ \\
\hline Random walk & $0.00005 \mathrm{deg} / \mathrm{sqrt}(\mathrm{s})$ & $0.000017 \mathrm{deg} / \mathrm{sqrt}(\mathrm{s})$ \\
\hline
\end{tabular}

performance which would probably necessitate screening of instruments by a vendor to achieve. The term "screening" refers to selection of units of higher than average quality from the production line. Although a "Medium Quality IMU" and a "High Quality IMU" might actually come from the same production line, the performance of the "High Quality IMU" would be significantly better than the performance of the "Medium Quality IMU" due to screening, but the cost would also be expected to be significantly greater.

The error models for the "Medium Quality IMU" and the "High Quality IMU" are provided in Table 1. The key differences in the models are the bias and scale factor values for the gyros and accelerometers and the gyro random walk. As will be discussed below, the gyro parameters are of particular importance in meeting the wedge angle requirement. Both IMUs are assumed to be strapdown units.

Table 1 - IMU Error Models

\section{Analysis Tools}

The majority of the analysis performed for this task was done using covariance analysis tools. The analysis was done in an inertial frame using the previously discussed reference trajectory and the error models for the Medium and High Quality IMUs. Errors in navigated position and velocity and small angle attitude errors were propagated using state space error equations of the following form 


$$
\begin{aligned}
& \delta \dot{R}=\delta V \\
& \delta \dot{V}=(\nabla g) \delta R+(s k e w[S F]) \delta \theta+C_{b}^{i} A B-C_{b}^{i}\left[\begin{array}{ccc}
S F^{B_{1}} & 0 & 0 \\
0 & S F^{B_{2}} & 0 \\
0 & 0 & S F^{B_{3}}
\end{array}\right] A S F E+\ldots \\
& \delta \dot{\theta}=-C_{b}^{i} B D-C_{b}^{i}\left[\begin{array}{ccc}
\omega_{b}(1) & 0 & 0 \\
0 & \omega_{b}(2) & 0 \\
0 & 0 & \omega_{b}(3)
\end{array}\right] G S F E+\ldots
\end{aligned}
$$

where $\mathrm{SF}^{\mathrm{B}}$ is the specific force transformed to the body frame and $\omega^{\mathrm{B}}$ is the angular velocity of the vehicle in the body frame. The measurement equations corresponding to various measurements types associated with the Navigation options under consideration are discussed below.
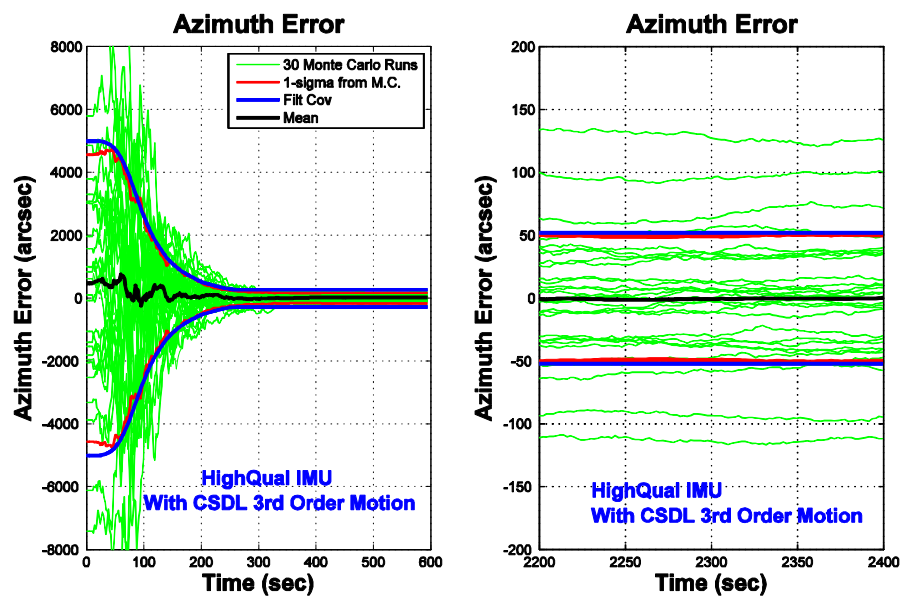

A limited amount of Monte Carlo analysis was performed as a check on the covariance analysis results. Because the error models are linear, the only significant nonlinearity in the system is the gravity feedback effect in which a position error results in a velocity error. As indicated in the representative plots of Figure 3, the Monte Carlo results in general correspond well to the covariance results. As a consequence, the covariance results were considered validated and appropriate for use in drawing conclusions.

Figure 3 - Representative Monte Carlo Results

\section{Pre-Launch And Post-Launch Error Contributions}

A point which arose early in the analysis was that, given the short flight time of the Ares 1, the errors which exist at launch have significantly greater effect on meeting the second stage MECO requirements than the errors accrued during powered flight. The covariance results in Table 2 illustrate this point. When initial attitude error is zero about each of the three axes, semi-major axis error at second stage MECO is well within the requirement and the wedge angle error is $18 \operatorname{arcsec}$ - well within the allocation of 84 arcsec. The semi-major axis is largely a result of accelerometer error and the wedge angle error is the result of attitude error developed during powered flight. When initial attitude errors of 10 arcsec about the horizontal North and East axes are present, the wedge angle error increases to approximately 19 arcsec with the semi-major axis error essentially unchanged. However, when an 80 arcsec initial attitude error about Down (a launch azimuth error) is added, the wedge angle reaches a corresponding value of approximately 80 arcsec. Again, the semi-major axis error is essentially the same because the accelerometer parameters are unchanged. These results lead to two conclusions. First, the wedge angle requirement will be significantly more difficult to meet than the semi-major axis requirement. Second, the driving error source in meeting the wedge angle requirement will be initial azimuth error. Consequently, the major issue in developing a Navigation architecture for Ares 1 becomes defining a method in which the initial value of launch azimuth can be accurately determined. 


\begin{tabular}{|c|c|c|c|c|}
\hline $\begin{array}{c}\text { Initial attitude } \\
\text { error about } \\
\text { Down }\end{array}$ & $\begin{array}{c}\text { Initial attitude } \\
\text { error about } \\
\text { North }\end{array}$ & $\begin{array}{c}\text { Initial attitude } \\
\text { error about } \\
\text { East }\end{array}$ & $\begin{array}{c}\text { Semi-major axis } \\
\text { error at MECO } \\
\text { (Medium IMU) }\end{array}$ & $\begin{array}{c}\text { Wedge angle } \\
\text { error at MECO } \\
\text { (Medium IMU) }\end{array}$ \\
\hline $80 \operatorname{arcsec}$ & $10 \operatorname{arcsec}$ & $10 \operatorname{arcsec}$ & $4980 \mathrm{ft}$ & $80 \operatorname{arcsec}$ \\
\hline $0 \operatorname{arcsec}$ & $10 \operatorname{arcsec}$ & $10 \operatorname{arcsec}$ & $4950 \mathrm{ft}$ & $19 \operatorname{arcsec}$ \\
\hline $0 \operatorname{arcsec}$ & $0 \operatorname{arcsec}$ & $0 \operatorname{arcsec}$ & $4885 \mathrm{ft}$ & $18 \operatorname{arcsec}$ \\
\hline
\end{tabular}

Table 2 - MECO Error As A Function Of Initial Attitude Error

\section{Leveling And Gyrocompassing}

The principle method for self-alignment of a Navigation system is the use of leveling and gyrocompassing. Because the determination of initial attitude has been shown to be the key factor in meeting the wedge angle requirement, a brief discussion of gyrocompassing and the contributing errors is considered next. Given accurate time, the orientation of the Earth with respect to the stars (inertial space) can be determined. This information usually takes the form of an inertial frame to Earth frame transformation matrix, which is defined by three rotation angles. If one can then determine attitude relative to the Earth at a given time (in the form of a body to Earth frame transformation matrix, again defined by three angles), attitude relative to inertial space can be determined by combining the two transformation matrices. Accomplishing this is a useful way of initializing an inertial Navigation system which departs from the surface of the Earth.

Since the inertial to Earth frame transformation is known given the time, the items to be determined are the three angles which represent the orientation of the Navigation system (body frame) with respect to an Earth fixed coordinate frame and thus define the body to Earth frame transformation matrix. If the "Down" direction can be determined, this will establish two of the three necessary angles. If "North" can then be determined, the third angle will be known. The process of determining "Down" is known as leveling and the process of determining "North" is called gyrocompassing.

For purposes of discussion, consider a Navigation system which includes an IMU with three orthogonal accelerometers and the three orthogonal gyros, with the gyro and accelerometer axes aligned. The launch site location and gravitational parameters are assumed known through a survey. If one of the accelerometers is pointed "Down" (or "Up"), the other two accelerometers should be normal to the gravity vector and thus should read zero. By physically (not common) or mathematically (more common) moving the platform (body frame) until two of the accelerometers read zero, the "Down" direction and thus two of the three required angles can be determined. This procedure generally works well and converges to an answer within a few seconds. The primary source of error is accelerometer bias. If an accelerometer reads a certain value with no input, it will actually have to be "tilted" slightly relative to the gravity vector such that a small component of gravity balances the bias and thus produces a zero output. Thus the leveling error in each of the two angles can be thought of as

$$
\text { LevelingError }=\operatorname{Arctan}\left(\frac{\text { AccelerometerBias }}{g}\right)
$$

Gyrocompassing works in a similar way, but instead of working with the gravity vector, the rotation rate vector of the Earth is used. For simplicity, imagine a Navigation system at the equator with the instrument axes pointed approximately North, East, and Down. Note that the North axis should nominally be aligned with the rotation rate vector of the Earth. In this case, the North gyro should read Earth rate and the East gyro should read zero. Thus one could physically or mathematically rotate the instrument axes about "Down" until the East gyro reads zero, at which point the North gyro axis should be pointing North. As before, if the primary instrument, in this case the East gyro, has a bias error such that it produces an output when it should see zero, the instrument axes will

American Institute of Aeronautics and Astronautics 092407 
need to be rotated slightly about "Down" such that a component of Earth rate offsets the East gyro bias. East gyro bias is thus the limiting factor in gyrocompassing accuracy. Note that because the rate of rotation of the Earth is small and thus may be difficult to observe in the presence of instrument noise, gyrocompassing may take many minutes to converge. The theoretical limit of gyrocompassing accuracy is a function of the equivalent East gyro drift and the latitude of the vehicle prior to launch which is given by

$$
\text { GyrocompassingError }=\left(\frac{\text { EastGyroDrift }}{\omega_{e} \cos (\text { lat })}\right)
$$

where $\omega_{\mathrm{e}}$ is the rotation rate of the Earth. Using the error model for a more accurate "High Quality IMU", the theoretical gyrocompassing error limit is found to be approximately 47 arcsec one sigma. In fact, when other gyro error sources are included in the analysis, the achievable gyrocompassing accuracy becomes approximately 52 arcsec, which is within the wedge angle error allocation of 84 arcsec. Using the error model for a less accurate "Medium Quality IMU", the theoretical gyrocompassing error is found to be 114 arcsec one sigma, which exceeds the wedge angle of requirement of 84 arcsec.

The pre-launch leveling and gyrocompassing procedure may be incorporated into the state space covariance analysis through the use of Zero Velocity Updates (ZUPTs). A Zero Velocity Update represents an acknowledgement that the vehicle is standing still with respect to the Earth. Because initial position is well known at a surveyed launch site, the inertial velocity due to Earth rate can be accurately determined. Any difference between this calculated value and the value reported by the IMU can be attributed to IMU error due to a combination of instrument error and alignment error. The effect of instrument error is comparatively small with respect to the effect of initial alignment error, thus allowing the estimation of the alignment error. The measurement equation used for a ZUPT in covariance analysis is

$$
z=-C_{i}^{g} \omega_{e} \times \delta R+C_{i}^{g} \delta V+\text { noise }
$$

where $\omega_{\mathrm{e}}$ is Earth rate in the inertial frame and $\mathrm{C}_{\mathrm{i}}{ }^{\mathrm{g}}$ is the transformation matrix from the inertial to the geodetic frame.

\section{Ascent Navigation Options}

Motivated by the realization that the determination of initial azimuth error is the driving factor in meeting the wedge angle requirement at MECO, six possible Ares 1 architectures were investigated. These were

a.) Use of external alignment as done in Apollo

b.) Use of a multi-position gyrocompassing algorithm similar to Shuttle

c.) Blending of data from multiple IMUs

d.) Use of GPS during ascent to determine alignment error

e.) Use of GPS during prelaunch to assist gyrocompassing

f.) Use of an IMU of sufficiently high quality

Each option is now considered in more detail.

\section{A. Optical Alignment As In Apollo}

The Saturn launch vehicles used a gimbaled IMU, supplemented by a theodolite to provide azimuth information prior to launch. From Reference 1,

The carefully controlled alignment of the ST-124 platform did not take place until the final events of the launch countdown. The procedure called for a precisely sited theodolite not far from the launch pad to aim a beam of light through a small opening in the IU high above the ground. The beam passed through a small window in the guidance platform where a pair of platform prisms reflected the beam back to the theodolite. Coated to work with two different wavelengths, the prisms aided in aligning the platform to its launch azimuth; when proper alignment was achieved, the acquisition light signal notified the mission control 
center. The integral orientation of the platform was modified based on the data obtained from the theodolite such that initial alignment was obtained.

\section{From Reference 2,}

The fixed prism error signal is transmitted from the theodolite through contact $\mathrm{A}$ to the $\mathrm{Y}$ gyro alignment loop, which will position the inner gimbal to the reference azimuth. At the same time, the served prism error signal from the theodolite will drive the servomotor on the inner gimbal to align the movable prism along the same baseline.

Use of an external optical alignment device was considered for Ares 1. Typically IMU vendors calibrate their units at the factory by first placing the unit of a rate table and accurately determining its orientation using an optical device mounted on the unit's case. The rate table can then be rotated about each of its three axes to allow the separation of various error sources, with the calculated values of these error sources used in algorithms within the IMU to remove the measured components of error and thus improve the effective performance of the IMU. The calibration values often drift with time and thus an IMU is considered within specifications for only a fixed period of time after calibration. The same optical device used for alignment of an IMU on a rate table during calibration can also be used in an external alignment procedure on the launch pad.

Theodolites are very accurate devices and alignment within 84 arcsec under laboratory conditions would not be an issue. For Ares 1, however, practical difficulties must also be considered. First, external access to the IMU is necessary. This means that a window or door in the vehicle is required. In addition, the Ares 1 baseline architecture consists of three identical IMU/flight computer strings as opposed to the single IMU on a Saturn vehicle. Examination of the layout of the instrument ring indicated that providing external line of sight access from the ground to three IMUs was prohibitive. Access to a theodolite mounted on the launch tower was considered, but alignment of the launch tower and motion of the tower and launch vehicle during alignment then come into play. Consequently, use of an external optical device was determined to have the potential for mitigating the azimuth alignment problem, but entailed significant practical drawbacks.

\section{B. Use Of Two Position Gyrocompass As In Shuttle}

The Shuttle also utilizes a gimbaled IMU, but uses a two-position gyrocompassing technique for initial azimuth alignment. From Reference 3,

In the gyrocompass alignment, each IMU is oriented so that the desired relative skew is achieved when the platforms are at their alignment orientation. During this phase, the IMUs are placed in two orientations relative to the north-west-up coordinate system. These two orientations differ only in a 90-degree rotation about the up axis.
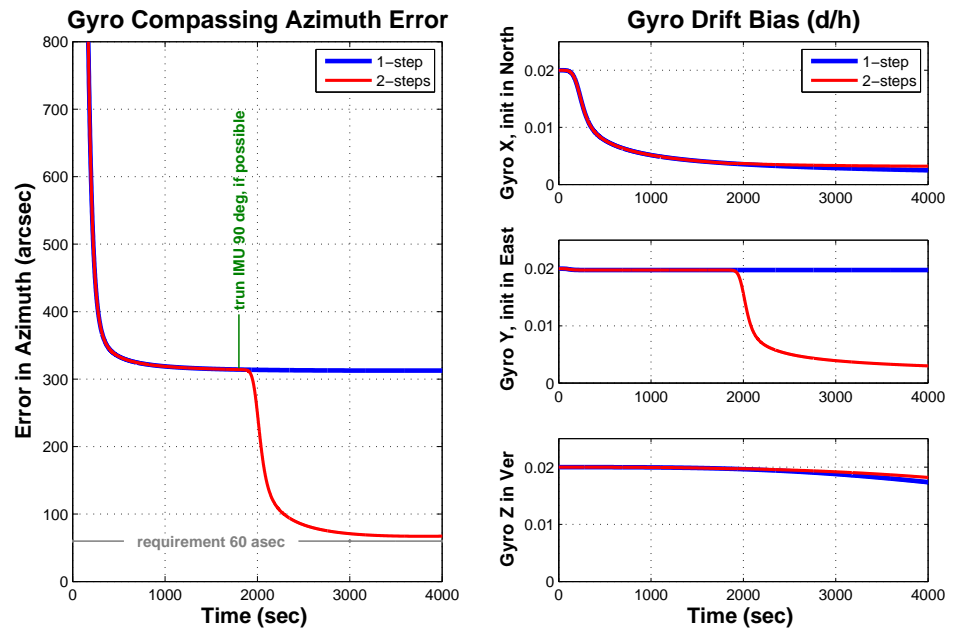

Figure 4 - Two Position Gyrocompass
The benefit of two position gyrocompassing is that the drift of the North gyro can first be accurately determined. The North gyro can then be rotated 90 degrees about the vertical such that it is directed East, at which point gyrocompassing is performed with an accurately calibrated gyro. This procedure is illustrated in Figure 4. Note from the plots that when the North gyro is rotated to East, the azimuth error of the two position gyrocompass drops significantly below the value of the one position gyrocompass. 
Use of two position gyrocompassing would be an effective solution for Ares 1 if the IMUs to be used were gimbaled. Because use of strapdown IMUs is planned, implementation of a two position gyrocompass would involve the addition of single axis gimbals around the strapdown IMUs. This could be done, and would in fact have the additional benefit of allowing partial calibration of the IMUs on the launch pad. However, the additional complexity and added weight, particularly when used with three IMUs, make this a less than ideal solution to the azimuth alignment problem.

\section{Blending Data From Multiple Medium Quality IMUs}

Gyrocompassing with a High Quality IMU was previously shown to meet the initial azimuth requirement necessary to meet the MECO wedge angle requirement, while gyrocompassing with a Medium Quality IMU was shown to lead to unacceptable initial azimuth error (Reference?). However, as a result of the Fail-Op/ Fail-Safe redundancy requirement, Ares will carry three IMUs. An

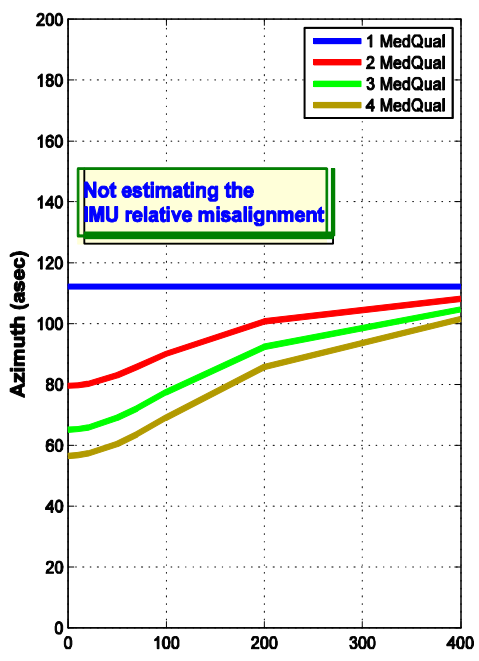

Relative misalignment (arcsec) analysis of combining data from multiple IMUs was performed to determine if the wedge angle requirement could be met. One complication to this approach is the existence of relative misalignments between the multiple units, a fact which degrades the achievable accuracy of the approach. As indicated in Figure 5, data from two, three, and four Medium Quality IMUs were combined for various values of relative misalignment. For a relative misalignment of 100 arcsec or less, combining data from three or four Medium Quality IMUs will meet the initial azimuth goal of 84 arcsec.

Based on this analysis, combination of data from multiple IMUs is a viable option. As with most options, however, there are drawbacks to contend with. Primary on the list of concerns with this approach is the calibration and stability of the misalignments between IMUs. An onpad alignment procedure could prove as difficult as the use of a theodolite to align the IMUs themselves, and an offpad alignment procedure leads to concerns about stability

Figure 5 - Use Of Data From Multiple IMUs of the misalignments during transport. Additional difficulties are incurred if an IMU assembly must be replaced on the pad. Of the options thus far considered, the combination of data from multiple IMUs appears most feasible, though concerns exist.

\section{Use Of GPS During Ascent}

The initial Ares 1 attitude error will quickly create velocity and position errors through the coupling of attitude error into velocity as indicated in the covariance equations above. As such, the correlations between position, velocity, and attitude may be used to correct attitude error if accurate measures of position and velocity are available after launch. GPS provides the very accurate measures of position and velocity required, and as such GPS may be used in ascent to determine attitude error. As indicated in Figure 6, the azimuth error associated with a single Medium Quality IMU is approximately 120 arcsec at launch. This error is brought within the necessary $84 \operatorname{arcsec}$ within a minute after launch if range and delta range measurements are available. If only range measurements

Americ:
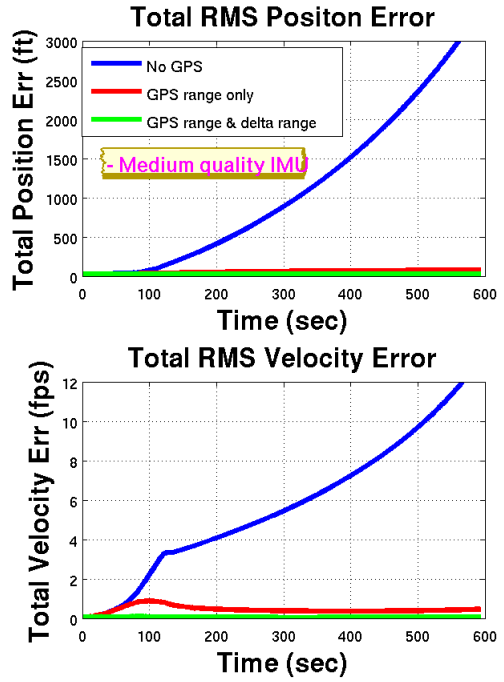

092407

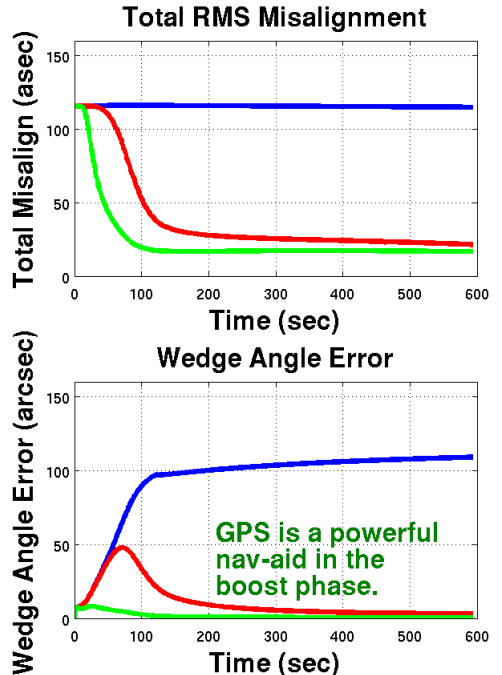


are available, the 84 arcsec goal is still met within two minutes after launch.

The use of GPS provides a practical solution to the initial azimuth issue, with the additional benefit of improved position and velocity information. From an accuracy point of view GPS is outstanding. However, use of GPS does require that the vehicle have an antenna and a receiver on board. In fact, Range Safety will have this hardware on board as a part of their instrumentation. If signals from the Range Safety instrumentation are available, use of GPS presents a highly beneficial enhancement to the Navigation function. However, when GPS is part of Range Safety it is not a flight critical component of the system due to redundancy of the Range safety instrumentation. Use of GPS by Navigation to address the initial azimuth issue would make GPS a flight critical component. In addition, the vehicle will need to steer out the azimuth error in flight, which could result in a small fuel penalty. Visibility of a sufficient number of satellites might also be a concern. All in all, however, GPS is an attractive option.

\section{E. Use Of GPS During Pre-Launch}

If GPS were used only prior to launch, it would not be a flight critical function because launch could be delayed if GPS was not available. The question is then whether sufficient benefit could be derived from use of GPS only on the launch pad. Because correlations between azimuth error and the quantities measured by GPS do not develop until sufficient specific force is applied in ascent, use of GPS on the launch pad will not significantly lower the achievable azimuth error at launch. The use of GPS can be shown to speed convergence through the measurement of vehicle motion during the alignment process, however, as indicated in Figure 7.
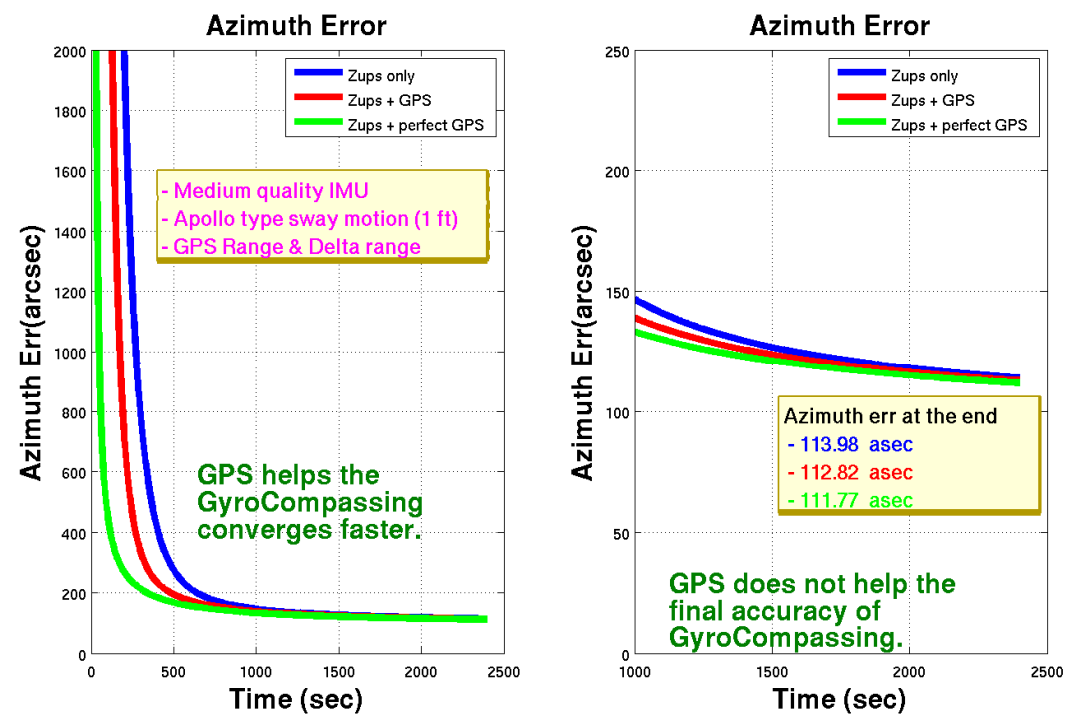

Figure 7 - Use Of GPS During Pre-Launch

\section{F. Use Of A High Quality IMU}

The most straightforward method of addressing initial Ares 1 alignment is procurement of an IMU of sufficient quality that gyrocompassing meets the 84 arcsec requirement. As shown previously, use of a High Quality IMU provides better than necessary performance, providing a theoretical initial azimuth error of $52 \mathrm{arcsec}$. The question is then how much can the High Quality IMU specification be relaxed such that the 84 arcsec requirement is still met. Consequently, an analysis of the effects of screening instruments to various levels was performed. Table 3 shows the wedge angle and semi-major axis errors achievable when the gyro drift and gyro random walk error values are ramped linearly between the medium and High Quality IMUs. As the gyro error parameters are reduced, the wedge angle error is correspondingly reduced. The semi-major axis error in Table 3 is largely insensitive to the changes in the gyro error parameters and is significantly less than that in Table 2 because the gyrocompassing procedure provides improvement to the accelerometer channels through the estimation of accelerometer scale factor in the vertical direction. Note that because screening to higher and higher quality involves moving further and further into the tails of a normal distribution of IMU performance, the cost effect of linearly increasing performance is nonlinear. 
Specifically, an IMU with performance half way between the Medium Quality IMU and the High Quality IMU would be expected to require less than half of the cost increase between the two units. As a result, even a modest reduction in the specifications of the High Quality IMU can result in significant cost savings.

\begin{tabular}{|c|c|c|c|}
\hline Gyro Drift & $\begin{array}{c}\text { Gyro Random } \\
\text { Walk }\end{array}$ & $\begin{array}{c}\text { Semi-major axis } \\
\text { error at MECO } \\
\text { (Medium IMU) }\end{array}$ & $\begin{array}{c}\text { Wedge angle error } \\
\text { at MECO } \\
\text { (Medium IMU) }\end{array}$ \\
\hline $0.006 \mathrm{deg} / \mathrm{hr}$ & $0.0030 \mathrm{deg} / \mathrm{sqrt}(\mathrm{hr})$ & $1499 \mathrm{ft}$ & $108 \mathrm{arcsec}$ \\
\hline $0.005 \mathrm{deg} / \mathrm{hr}$ & $0.0027 \mathrm{deg} / \mathrm{sqrt}(\mathrm{hr})$ & $1441 \mathrm{ft}$ & $93 \mathrm{arcsec}$ \\
\hline $0.004 \mathrm{deg} / \mathrm{hr}$ & $0.0023 \mathrm{deg} / \mathrm{sqrt}(\mathrm{hr})$ & $1386 \mathrm{ft}$ & $77 \mathrm{arcsec}$ \\
\hline $0.003 \mathrm{deg} / \mathrm{hr}$ & $0.0020 \mathrm{deg} / \mathrm{sqrt}(\mathrm{hr})$ & $1343 \mathrm{ft}$ & $62 \mathrm{arcsec}$ \\
\hline
\end{tabular}

Table 3 - MECO Error For A Range Of Values Of Gyro Drift And Gyro Random Walk With Other Parameters Corresponding To A Medium Quality IMU

From Table 3, an IMU with gyro drift of $0.004 \mathrm{deg} / \mathrm{hr}$ and $0.0023 \mathrm{deg} / \mathrm{sqrt}(\mathrm{hr})$ will meet the 84 arcsec wedge angle requirement as well as the semi-major axis requirement of 16,223 feet. As such, the desired gyrocompassing accuracy can be achieved with an IMU whose parameters are a compromise between the High and Medium Quality IMUs. Such a solution is straightforward, having no need for additional instrumentation, hardware, or special procedures, with the primary concern being cost of procuring relatively high quality IMUs.

\section{Conclusion}

Based on the amount of additional hardware required and the associated increase in weight and complexity, the use of external optical alignment devices and the use of gimbals to implement a two position gyrocompass were eliminated from consideration for Ares 1 early in the analysis cycle. The use of GPS, though attractive, raised challenges given the additional hardware, interfaces, and processing which would be required. The remaining candidates were then the use of an IMU of sufficient quality to meet the gyrocompassing requirement on its own and the combination of data from multiple Medium Quality IMUs. Ultimately, the Ares I program must weigh the complexity associated with the blending of data from multiple IMUs against the likely additional cost associated with a higher quality IMU.

\section{References}

The following references were cited in the text.

1.) http://history.nasa.gov/SP-4206/ch8.htm

2.) Haeussermann, Walter and Robert Clifton Duncan, Status Of Guidance And Control Methods, Instrumentation, And Techniques As Applied In The Apollo Project,

3.) http://spaceflight.nasa.gov/shuttle/reference/shutref/orbiter/avionics/gnc/imu.html 